\title{
Permanence and Global Stability for a Non-Autonomous Predator-Prey Model with Modified Leslie-Gower and Holling-Type II Schemes with Delays
}

\author{
Lin Hu, Linfei Nie \\ College of Mathematics and Systems Science, Xinjiang University, Urumqi, China \\ E-mail: Ifnie@163.com \\ Received August 18, 2010; revised November 11, 2010; accepted November 14, 2010
}

\begin{abstract}
In this paper, a nonautonomous predator-prey system based on a modified version of the Leslie-Gower scheme and Holling-type II scheme with delayed effect is investigated. The general criteria of integrable form on the permanence are established. By constructing suitable Lyapunov functionals, a set of easily verifiable sufficient conditions are derived for global stability of any positive solutions to the model.
\end{abstract}

Keywords: Predator-Prey System, Leslie-Gower and Holling-Type-II Functional Response, Permanence, Global Stability

\section{Introduction}

Predator-prey behavior is a form of very common biological interaction in nature. There are many mathematical models to model predator-prey behavior such as Lotka-Volterra system, Chemostat-type system, Kolmogorov system, etc (see [1-6]). In recent years there has been a growing interest in the study of mathematical models incorporates a modified version of Leslie-Gower functional response as well as that of the Holling-type II (see [7-9]). In particular, in [10] the authors consider the following model

$$
\left\{\begin{array}{l}
\frac{\mathrm{d} x(t)}{\mathrm{d} t}=x(t)\left[a-b x(t)-\frac{c y(t)}{x(t)+k_{1}}\right] . \\
\frac{\mathrm{d} y(t)}{\mathrm{d} t}=y(t)\left[d-\frac{e y(t)}{x(t)+k_{2}}\right]
\end{array}\right.
$$

This two species food chain model describes a prey population $x$ which serves as food for predator $y, a, b, c$, $e, k_{1}$ and $k_{2}$ are positive parameters. They established the sufficient conditions for the boundedness, existence of a positively invariant attracting set and global stability of coexisting interior equilibrium. In [11] the authors considered the dynamical behavior of system (1.1) with delays, and establish the sufficient conditions for the existence positive equilibrium, permanence and global stability of positive equilibrium. The dynamical behavior of system (1.1) also has been discussed by many authors (see, for example, [7,12] and the references cited therein).

However, we note that any biological or environmental parameters are naturally subject to fluctuation in time. As [13] pointed out that the growth properties of every natural population vary through time. Most, and perhaps all, of this variation arises ultimately from fluctuations in the population's environment. Physical environmental conditions usually change greatly through the year and can influence organisms directly. Good weather can stimulate growth in body size and reproduction, and bad weather can cause death. Similarly, the biological environment can fluctuate in ways that influence population dynamics. These kinds of time variation in population dynamical events can exert profound effects on the ecology and evolution of individual species and on the composition of ecological communities.

In this paper, we are concerned with the effects of the time-dependent of ecological and environmental parameters and time delays due to gestation and negative feedbacks on the global dynamics of predator-prey systems with Modified Leslie-Gower and Holling-Type II Schemes. Therefore, we consider the following delayed differential system:

$$
\left\{\begin{array}{l}
\frac{\mathrm{d} x_{1}(t)}{\mathrm{d} t}=x_{1}(t)\left[r_{1}(t)-b_{1}(t) x_{1}\left(t-\tau_{1}\right)-\frac{a_{1}(t) x_{2}\left(t-\tau_{2}\right)}{x_{1}\left(t-\tau_{1}\right)+k_{1}(t)}\right] \\
\frac{\mathrm{d} x_{2}(t)}{\mathrm{d} t}=x_{2}(t)\left[r_{2}(t)-\frac{a_{2}(t) x_{2}\left(t-\tau_{4}\right)}{x_{1}\left(t-\tau_{3}\right)+k_{2}(t)}\right]
\end{array}\right.
$$


with initial conditions

$$
\begin{gathered}
x_{i}(\theta)=\phi_{i}(\theta), \quad \theta \in[-\tau, 0], \\
\phi_{i}(\theta) \in C_{+}[-\tau, 0], i=1,2, \tau=\max \left\{\tau_{1}, \tau_{2}, \tau_{3}, \tau_{4}\right\},
\end{gathered}
$$

where $C_{+}[-\tau, 0]:=\{\phi \in C[-\tau, 0]: \phi(s) \geq 0, s \in[-\tau, 0)$, and $\phi(0)>0\}, x_{1}(t)$ and $x_{2}(t)$ denote the densities of prey and predator population, respectively; $\tau_{i} \geq 0$ $(i=1,2,3,4)$ denote the time delays due to negative feedbacks of the prey and the predator population, $b_{1}(t), r_{i}(t), a_{i}(t)$ and $k_{i}(t)(i=1,2)$ are model parameters. These parameters are defined as follows: $r_{1}(t)$ is the growth rate of prey $x_{1}, b_{1}(t)$ measures the strength of competition among individuals of species $x_{1}, a_{1}(t)$ is the maximum value which per capita reduction rate of $x_{1}$ can attain, $k_{1}(t)$ and $k_{2}(t)$ measure the extent to which environment provides protection to prey $x_{1}$ and predator $x_{2}$, respectively; $r_{2}(t)$ is the growth rate of predator $x_{2}$, and $a_{2}(t)$ has a similar meaning to $a_{1}(t)$.

The organization of this paper is as follows. In the next section, we present some basic assumptions for system (1.2) and two important lemmas on the nonautonomous single-species logistic system. In Section 3, we will state and prove the sufficient conditions of integrable form on the permanence of solutions for system (1.2). We also by means of suitable Lyapunov functionals, a set of easily verifiable sufficient conditions are derived for global stability of any positive solutions of system (1.2). Numerical result is presented to illustrate the validity of our main results.

\section{Preliminaries}

Let $R_{+0}:=[0, \infty)$ and $R_{+}:=(0, \infty)$. For a bounded continuous function $g(t)$ on $R$, we use the following notations: $g^{u}:=\sup _{t \in R} g(t), \quad g^{l}:=\inf _{t \in R} g(t)$.

For system (1.2), we introduce the following assumptions.

$\left(\mathrm{H}_{1}\right)$ Function $b_{1}(t), r_{i}(t), a_{i}(t), k_{i}(t)$ are continuous and bounded on $R_{+0}, a_{1}(t) \geq 0$ for all $t \geq 0$, and $\inf _{t \geq 0} k_{i}(t)>0(i=1,2)$.

$\left(\mathrm{H}_{2}\right)$ There a constant $\mu>0$ such that

$$
\liminf _{t \rightarrow \infty} \int_{t}^{t+\mu} b_{1}(\tau) \mathrm{d} \tau>0, \liminf _{t \rightarrow \infty} \int_{t}^{t+\mu} a_{2}(\tau) \mathrm{d} \tau>0 .
$$

$\left(\mathrm{H}_{3}\right)$ There is a constant $\omega>0$ such that

$$
\liminf _{t \rightarrow \infty} \int_{t}^{t+\omega} r_{1}(\tau) \mathrm{d} \tau>0, \liminf _{t \rightarrow \infty} \int_{t}^{t+\omega} r_{2}(\tau) \mathrm{d} \tau>0 .
$$

It is well known by the fundamental theory of functional differential equations [5] that system (1.2) has a unique solution $x(t)=\left(x_{1}(t), x_{2}(t)\right)$ satisfying initial conditions (1.3). If $x_{i}(t)>0(i=1,2)$ on the interval of existence, then $x(t)$ is said to be a positive solution. It is easy to verify that solutions of system (1.2) corresponding to initial conditions (1.3) are defined on $[0, \infty)$ and remain positive for all $t \geq 0$.

We consider the following single-species nonautonomous logistic system with a parameter

$$
\frac{\mathrm{d} u}{\mathrm{~d} t}=u\left[r_{2}(t)-\frac{\left.a_{2}(t)\right) u}{\alpha+k_{2}(t)}\right]:=u g(t, u, \alpha) .
$$

Obviously, if Assumption $\left(\mathrm{H}_{1}\right)$ holds, then $g(t, u, \alpha)$ is a continuous function defined on

$$
(t, u, \alpha) \in R_{+0} \times R_{+} \times\left[0, \alpha_{0}\right],
$$

where $\alpha_{0}$ is constant. We easily prove that for any $\left(t_{0}, u_{0}\right) \in R_{+0} \times R_{+}$and $\alpha \in\left[0, \alpha_{0}\right]$ system (2.1) has a unique solution $u_{\alpha}(t)$ satisfying initial condition $u_{\alpha}\left(t_{0}\right)=u_{0}$. It is easy to see that $u_{\alpha}(t)$ is positive for all $t \geq t_{0}$ if the initial value $u_{0}>0$. If Assumptions $\left(\mathrm{H}_{1}\right)-\left(\mathrm{H}_{3}\right)$ hold, then the following statements can be prove to be true.

$\left(A_{1}\right)$ For any constant $\sigma>1, g(t, u, \alpha)$ is bounded on $R_{+0} \times\left[\sigma^{-1}, \sigma\right] \times\left[0, \alpha_{0}\right]$.

$\left(A_{2}\right)$ There are positive constants $k_{1}, k_{2}, \omega_{1}, \omega_{2}$ and $k_{1}<k_{2}$ such that

$$
\begin{aligned}
& \liminf _{t \rightarrow \infty} \int_{t}^{t+\omega_{1}} g\left(\tau, k_{1}, 0\right) \mathrm{d} \tau>0, \\
& \limsup _{t \rightarrow \infty} \int_{t}^{t+\omega_{2}} g\left(\tau, k_{2}, 0\right) \mathrm{d} \tau<0 .
\end{aligned}
$$

$\left(A_{3}\right)$ Partial derivative $\partial g(t, u, 0) / \partial u$ exists for all $(t, u) \in R_{+0} \times R_{+}$, and there are nonnegative continuous function $q(t)$ and a constant $\omega>0$, satisfying

$$
\liminf _{t \rightarrow \infty} \int_{t}^{t+\omega} q(\tau) \mathrm{d} \tau>0
$$

and a continuous function $p(u)$, satisfying $p(u)>0$ for all $u \in R_{+}$, such that

$$
\frac{\partial g(t, u, 0)}{\partial u} \leq-q(t) p(u) \text { for all }(t, u) \in R_{+0} \times R_{+} .
$$

$\left(A_{4}\right)$ Partial derivative $\partial g(t, u, \alpha) / \partial \alpha$ exists for all $(t, u, \alpha) \in R_{+0} \times R_{+} \times\left[0, \alpha_{0}\right]$, and for any constant $U>0$, $\partial g(t, u, \alpha) / \partial \alpha$ is also bounded on

$$
(t, u, \alpha) \in R_{+0} \times(0, U] \times\left[0, \alpha_{0}\right] .
$$

In system (2.1), when parameter $\alpha=0$ we obtain the following system

$$
\frac{\mathrm{d} u}{\mathrm{~d} t}=u\left[r_{2}(t)-\frac{a_{2}(t) u}{k_{2}(t)}\right]:=u g(t, u, 0)
$$

Let $u_{0}^{*}(t)$ be a fixed positive solution of system (2.2) defined on $R_{+0}$. We say that $u_{0}^{*}(t)$ is globally uniformly attractive on $R_{+0}$, if for any constants $\eta>1$ and $\varepsilon>0$ there is a constants $T(\eta, \varepsilon)>0$ such that 
for any initial time $t_{0} \in R_{+0}$ and any solution $u_{0}(t)$ of system (2.1) with $u_{0}\left(t_{0}\right) \in\left[\eta^{-1}, \eta\right]$, one has $\left|u_{0}(t)-u_{0}^{*}(t)\right|<\varepsilon$ for all $t \geq t_{0}+T(\eta, \varepsilon)$. By Lemma 1 given in [14], we have the following result.

Lemma 2.1 Suppose that Assumptions $\left(A_{1}\right)-\left(A_{3}\right)$ hold, then

a) There is a constants $M>1$ such that

$$
M^{-1} \leq \liminf _{t \rightarrow \infty} u_{0}(t) \leq \limsup _{t \rightarrow \infty} u_{0}(t) \leq M
$$

for any positive solution $u_{0}\left(t_{0}\right)$ of system (2.2).

b) Each fixed positive solution $u_{0}^{*}(t)$ of system (2.2) is globally uniformly attractive on $R_{+0}$

Let $u_{0} \in R_{+}, t_{0} \in R_{+0}$ and $\alpha \in\left[0, \alpha_{0}\right]$, and further let $u_{\alpha}(t)$ and $u_{0}(t)$ be the solutions of systems (2.1) and (2.2) with initial value $u_{\alpha}\left(t_{0}\right)=u_{0}$ and $u_{0}\left(t_{0}\right)=u_{0}$, respectively. By Lemma 2 given in [14], we further have the following result.

Lemma 2.2 Suppose that assumptions $\left(A_{1}\right)-\left(A_{4}\right)$ hold, then $u_{\alpha}(t)$ converges to $u_{0}(t)$ uniformly for $t \in\left[t_{0}, \infty\right)$ as $\alpha \rightarrow 0$.

\section{Main Results}

In this section, we proceed to discussion on the permanence and global stability of any positive solution of system (1.2) corresponding to initial conditions (1.3).

We first give the result of the ultimate boundedness of any solution for system (1.2).

Theorem 3.1 Suppose that Assumptions $\left(\mathrm{H}_{1}\right)-\left(\mathrm{H}_{3}\right)$ hold, then any solution $x(t)=\left(x_{1}(t), x_{2}(t)\right)$ of system (1.2) corresponding to initial conditions (1.3) are ultimately bounded.

Proof: Let $x(t)=\left(x_{1}(t), x_{2}(t)\right)$ be any solution of system (1.2) corresponding to initial conditions (1.3). From the first equation of system (1.2) we have

$$
\frac{\mathrm{d} x_{1}(t)}{\mathrm{d} t} \leq x_{1}(t) r_{1}(t) \leq r_{1}^{u} x_{1}(t) \text { for all } t \geq 0 \text {. }
$$

For any $t \geq \tau$ and $s \in[-\tau, 0]$, integrating (3.1) from $t+s$ to $t$ we obtain

$$
x_{1}(t+s) \geq x_{1}(t) \exp \left(r_{1}^{u} s\right) \geq x_{1}(t) \exp \left(-r_{1}^{u} \tau\right) .
$$

By assumptions $\left(\mathrm{H}_{1}\right)$, we further have

$$
\frac{\mathrm{d} x_{1}(t)}{\mathrm{d} t} \leq x_{1}(t)\left[r_{1}(t)-b_{1}(t) x_{1}(t) \exp \left(-r_{1}^{u} \tau\right)\right]
$$

for all $t \geq 0$. It is proved in many articles, for example, see [15], that under Assumptions $\left(\mathrm{H}_{1}\right)-\left(\mathrm{H}_{3}\right)$ any positive solution $u(t)$ of the following non-autonomous single-species logistic equation

$$
\frac{\mathrm{d} u(t)}{\mathrm{d} t}=u(t)\left[r_{1}(t)-b_{1}(t) u(t) \exp \left(-r_{1}^{u} \tau\right)\right]
$$

is ultimately bounded. Hence, using the comparison theorem, we can obtain that there is a constant $M_{1}>0$ such that for any solution $x(t)=\left(x_{1}(t), x_{2}(t)\right)$ of system (1.2) corresponding to initial conditions (1.3), there is a $t_{1} \geq 0$ such that $x_{1}(t)<M_{1}$ for all $t \geq t_{1}$.

From the second equation of system (1.2) we have

$$
\frac{\mathrm{d} x_{2}(t)}{\mathrm{d} t} \leq x_{2}(t) r_{2}(t) \leq r_{2}^{u} x_{2}(t) \text {. }
$$

For any $t \geq \tau$ and $s \in[-\tau, 0]$, integrating (3.2) from $t+s$ to $t$ we obtain

$$
x_{2}(t+s) \geq x_{2}(t) \exp \left(r_{2}^{u} s\right) \geq x_{2}(t) \exp \left(-r_{2}^{u} \tau\right)
$$

By assumptions $\left(\mathrm{H}_{1}\right)$, we further have

$$
\frac{\mathrm{d} x_{2}(t)}{\mathrm{d} t} \leq x_{2}(t)\left[r_{2}(t)-\frac{a_{2}(t) x_{2}(t)}{M_{1}+k_{2}(t)} \exp \left(-r_{2}^{u} \tau\right)\right]
$$

for all $t \geq t_{1}+\tau$. The comparison equation is the logistic equation

$$
\frac{\mathrm{d} y(t)}{\mathrm{d} t}=y(t)\left[r_{2}(t)-\frac{a_{2}(t) y(t)}{M_{1}+k_{2}(t)} \exp \left(-r_{2}^{u} \tau\right)\right] .
$$

Similarly, by Assumptions $\left(\mathrm{H}_{1}\right)-\left(\mathrm{H}_{3}\right)$, we further can obtain that there is constant $M_{2}>0$ such that for any solution $x(t)=\left(x_{1}(t), x_{2}(t)\right)$ of system (1.2) corresponding to initial conditions (1.3), there is a $t_{2} \geq t_{1}$ such that $x_{2}(t)<M_{2}$ for all $t \geq t_{2}$. Therefore, the solution $x(t)$ is ultimately bounded. This completes the proof of this theorem.

In particular, when parameter $\tau_{2}=\tau_{4}=0$ in system (1.2), we obtain the following system

$$
\left\{\begin{array}{l}
\frac{\mathrm{d} x_{1}(t)}{\mathrm{d} t}=x_{1}(t)\left[r_{1}(t)-b_{1}(t) x_{1}\left(t-\tau_{1}\right)-\frac{a_{1}(t) x_{2}(t)}{x_{1}\left(t-\tau_{1}\right)+k_{1}(t)}\right] \\
\frac{\mathrm{d} x_{2}(t)}{\mathrm{d} t}=x_{2}(t)\left[r_{2}(t)-\frac{a_{2}(t) x_{2}(t)}{x_{1}\left(t-\tau_{3}\right)+k_{2}(t)}\right]
\end{array}\right.
$$

with initial conditions

$$
x_{1}(\theta)=\phi_{1}(\theta), \theta \in\left[-\tau_{1}, 0\right], \phi_{1}(\theta) \in C_{+}[-\tau, 0], x_{2}(0)>0
$$

As a consequence of Theorem 3.1, we have the following corollary on the ultimate boundedness of any solution for system (3.3) with the initial conditions (3.4).

Corollary 3.1 Suppose that Assumptions $\left(\mathrm{H}_{1}\right)-\left(\mathrm{H}_{3}\right)$ hold, then any solution $x(t)=\left(x_{1}(t), x_{2}(t)\right)$ of system (3.3) corresponding to initial conditions (3.4) is ultimately bounded.

Next, on the permanence of component $x_{2}$ of system (1.2) with the initial conditions (1.3), we have the following result.

Theorem 3.2 Suppose that Assumptions $\left(\mathrm{H}_{1}\right)-\left(\mathrm{H}_{3}\right)$ hold, then the component $x_{2}$ of system (1.2) is permanent, in the sense there is a constant $\eta>0$ such that 
$\liminf _{t \rightarrow \infty} x_{2}(t)>\eta$ for all solutions of system (1.2) corresponding to initial conditions (1.3).

Proof: Let $x(t)=\left(x_{1}(t), x_{2}(t)\right)$ be any solution of system (1.2) corresponding to initial conditions (1.3). From Theorem 3.1, there is constant $M>0$ such that for any positive solution $x(t)$ of system (1.2), there is a $T \geq 0$ such that $x_{i}(t)<M(i=1,2)$ for all $t \geq T$. Therefore, from the second equation of system (1.2) we have

$$
\frac{\mathrm{d} x_{2}(t)}{\mathrm{d} t} \geq x_{2}(t)\left[r_{2}(t)-\frac{a_{2}(t) M}{k_{2}(t)}\right] \geq-\alpha_{1} x_{2}(t)
$$

for all $t \geq T+\tau$, where

$$
\alpha_{1}=\sup _{t \in R_{+}}\left\{\left|r_{2}(t)-a_{2}(t) M / k_{2}(t)\right|\right\} .
$$

For any $t \geq T+\tau$ and $s \in[-\tau, 0]$, integrating (3.5) from $t+s$ to $t$ we obtain

$$
x_{2}(t+s) \leq x_{2}(t) \exp \left(-\alpha_{1} s\right) \leq x_{2}(t) \exp \left(\alpha_{1} \tau\right) .
$$

Further, we have

$$
\frac{\mathrm{d} x_{2}(t)}{\mathrm{d} t} \geq x_{2}(t)\left[r_{2}(t)-\frac{a_{2}(t) x_{2}(t)}{k_{2}(t)} \exp \left(\alpha_{1} \tau_{2}\right)\right]
$$

for all $t \geq T+\tau$. By Assumptions $\left(\mathrm{H}_{1}\right)-\left(\mathrm{H}_{3}\right)$ and Lemma 2.1, we can obtain that the component $u$ of system

$$
\frac{\mathrm{d} u(t)}{\mathrm{d} t}=u(t)\left[r_{2}(t)-\frac{a_{2}(t) u(t)}{k_{2}(t)} \exp \left(\alpha_{1} \tau_{2}\right)\right]
$$

is permanent. Hence, using the comparison theorem, we can obtain the component $x_{2}$ of system (1.2) is permanent. This completes the proof of this theorem.

In order to obtain permanence of component $x_{1}$ of system (1.2), we consider the following auxiliary system with a parameter

$$
\frac{\mathrm{d} x_{2}(t)}{\mathrm{d} t}=x_{2}(t)\left[r_{2}(t)-\frac{a_{2}(t) x_{2}(t)}{\alpha+k_{2}(t)} \exp \left(-r_{2}^{u} \tau_{4}\right)\right] \text {. }
$$

In particular, when $\alpha=0$ in system (3.6), we obtain the following system

$$
\frac{\mathrm{d} x_{2}(t)}{\mathrm{d} t}=x_{2}(t)\left[r_{2}(t)-\frac{a_{2}(t) x_{2}(t)}{k_{2}(t)} \exp \left(-r_{2}^{u} \tau_{4}\right)\right] \text {. }
$$

By Assumptions $\left(\mathrm{H}_{1}\right)-\left(\mathrm{H}_{3}\right)$, we see that system (3.7) satisfies all conditions of Lemma 2.1. Hence, by Lemma 2.1, each positive of system (3.7) is globally asymptotically stable. Let $x_{20}^{*}(t)$ be some fixed solution of system (3.7) with initial value $x_{20}^{*}(0)>0$. On the permanence of component $x_{1}$ for system (1.2), we have the following result.

Theorem 3.3 Suppose that Assumptions $\left(\mathrm{H}_{1}\right)-\left(\mathrm{H}_{3}\right)$ hold and there is a constant $\lambda>0$ such that

$$
\liminf _{t \rightarrow \infty} \int_{t}^{t+\lambda}\left[r_{1}(s)-\frac{a_{1}(s)}{k_{1}(s)} x_{20}^{*}\left(s-\tau_{2}\right)\right] \mathrm{d} s>0,
$$

then the component $x_{1}$ of system (1.2) is permanence.

Proof: Let $x(t)=\left(x_{1}(t), x_{2}(t)\right)$ be any solution of system (1.2) corresponding to initial conditions (1.3). From Theorems 3.1 and 3.2, there are constants $M>0$ and $m>0$ such that for any positive solution $x(t)$ of system (1.2), there is a $T \geq 0$ such that $x_{1}(t)<M$ and $m<x_{2}(t)<M$ for all $t \geq T$.

In fact, if inequality (3.8) is true, then by Assumption $\left(\mathrm{H}_{3}\right)$, we can choose enough small positive constants $\varepsilon_{0}, \varepsilon_{1}, \delta$ and $\varepsilon_{0}>\varepsilon_{1}$, and an enough large $T_{0} \geq T$ such that

$$
\int_{t}^{t+\lambda}\left[r_{1}(s)-b_{1}(s) \varepsilon_{0}-\frac{a_{1}(s)}{k_{1}(s)}\left(x_{20}^{*}\left(s-\tau_{2}\right)+\varepsilon_{1}\right)\right] \mathrm{d} s>\delta
$$$$
\text { for all } t \geq T_{0}+\tau \text {. }
$$

For any $\alpha>0$, let $x_{2 \alpha}(t)$ be the solution of system (3.6) with initial value $x_{2 \alpha}(0)=x_{20}^{*}(0)$. Hence, by conclusion (b) of Lemma 2.1 and Lemma 2.2, there is a constant $\alpha_{0}>0$ such that

$$
x_{2 \alpha}(t)<x_{20}^{*}(t)+\frac{\varepsilon_{1}}{2}
$$

for all $t \geq 0$ and $\alpha \in\left[0, \alpha_{0}\right]$.

Let $\varepsilon=\min \left\{\alpha_{0}, \varepsilon_{1}\right\}, \quad x_{2 \varepsilon}(t)$ be any positive solution of the following system

$$
\frac{\mathrm{d} x_{2}(t)}{\mathrm{d} t}=x_{2}(t)\left[r_{2}(t)-\frac{a_{2}(t) x_{2}(t)}{\varepsilon+k_{2}(t)} \exp \left(-r_{2}^{u} \tau_{4}\right)\right]
$$

with initial value $x_{2 \varepsilon}(0)=x_{20}^{*}(0)$.

In the following, we will use two claims to complete the proof of Theorem 3.3.

Claim 3.1 For the above constant $\varepsilon$, there always exist $\limsup _{t \rightarrow \infty} x_{1}(t)>\varepsilon$ for any positive solution $x(t)$ of system (1.2).

In fact, if Claim 3.1 is not true, then there is a positive solution $x(t)=\left(x_{1}(t), x_{2}(t)\right)$ of system (1.2) such that $\limsup _{t \rightarrow \infty} x_{1}(t) \leq \varepsilon$. Hence, there is a $T_{1} \geq T_{0}$ such that $x_{1}(t)<\varepsilon$ for all $t \geq T_{1}$. Further, using the comparison theorem and Lemma 2.1, we can obtain that there is a constant $T_{2} \geq T_{1}$ such that

$$
x_{2}(t) \leq x_{2 \varepsilon}(t)+\frac{\varepsilon_{1}}{2}<x_{20}^{*}(t)+\varepsilon_{1}
$$

for all $t \geq T_{2}+\tau$. From the first equation of system (1.2) we have

$$
\begin{aligned}
& x_{1}(t) \geq x_{1}\left(T_{2}+\tau\right) \\
& x \exp \int_{T_{2}+\tau}^{t}\left[r_{1}(s)-b_{1}(s) \varepsilon-\frac{a_{1}(s)}{k_{1}(s)}\left(x_{2}^{*}\left(s-\tau_{2}\right)+\varepsilon_{1}\right)\right] \mathrm{d} s
\end{aligned}
$$


for all $t \geq T_{2}+\tau$. By (3.9) it follows that $x_{1}(t) \rightarrow \infty$ as $t \rightarrow \infty$. This is contradictory with $x_{1}(t)<\varepsilon$ for all $t \geq T_{2}+\tau$. From this contradiction we finally conclude that

$$
\limsup _{t \rightarrow \infty} x_{1}(t)>\varepsilon
$$

Therefore, Claim 3.1 is true.

Claim 3.2 There is a constant $\eta>0$ such that $\liminf _{t \rightarrow \infty} x_{1}(t)>\eta$ for any positive solution $x(t)$ of system (1.2).

If Claim 3.2 is not true, then there is a sequence of function

$$
\left\{\Phi_{n}=\left(\phi_{1 n}, \phi_{2 n}\right): \phi_{i n} \in C_{+}[-\tau, 0], i=1,2\right\}
$$

such that for the solution $\left(x_{1}\left(t, \Phi_{n}\right), x_{2}\left(t, \Phi_{n}\right)\right)$ of system (1.2)

$$
\liminf _{t \rightarrow \infty} x_{1}\left(t, \Phi_{n}\right)<\frac{\varepsilon}{n^{2}}, n=1,2, \cdots .
$$

By Claim 3.1, for every $n$ there are two time sequences $\left\{s_{q}^{(n)}\right\}$ and $\left\{t_{q}^{(n)}\right\}$, satisfying

$$
0<s_{1}^{(n)}<t_{1}^{(n)}<s_{2}^{(n)}<t_{2}^{(n)}<\cdots<s_{q}^{(n)}<t_{q}^{(n)}<\cdots
$$

and $\lim _{q \rightarrow \infty} s_{q}^{(n)}=\infty$, such that

$$
x_{1}\left(s_{q}^{(n)}, \Phi_{n}\right)=\frac{\varepsilon}{n}, x_{1}\left(t_{q}^{(n)}, \Phi_{n}\right)=\frac{\varepsilon}{n^{2}}
$$

and

$$
\frac{\varepsilon}{n^{2}}<x_{1}\left(t, \Phi_{n}\right)<\frac{\varepsilon}{n} \quad \text { for all } \quad t \in\left(s_{q}^{(n)}, t_{q}^{(n)}\right)
$$

From the ultimate boundedness of system (1.2) and Theorem 3.2, we can choose a positive constant $T^{(n)}$ for every $n$ such that $x_{1}\left(t, \Phi_{n}\right)<M$ and $m<x_{2}\left(t, \Phi_{n}\right)<M$ for all $t>T^{(n)}+\tau$. Further, there is an integer $K_{1}^{(n)}>0$ such that $s_{q}^{(n)}>T^{(n)}+\tau$ for all $q>K_{1}^{(n)}$. Let $q>K_{1}^{(n)}$, then for any $t \in\left[s_{q}^{(n)}, t_{q}^{(n)}\right]$ we have

$$
\begin{aligned}
\frac{\mathrm{d} x_{1}\left(t, \Phi_{n}\right)}{\mathrm{d} t} & \geq x_{1}\left(t, \Phi_{n}\right)\left[r_{1}(t)-b_{1}(t) M-\frac{a_{1}(t) M}{k_{1}(t)}\right] \\
& \geq-\gamma_{0} x_{1}\left(t, \Phi_{n}\right),
\end{aligned}
$$

where $\gamma_{0}=\sup _{t \geq 0}\left[r_{1}(t)+b_{1}(t) M+a_{1}(t) / k_{1}(t) M\right]$. Integrating the above inequality from $s_{q}^{(n)}$ to $t_{q}^{(n)}$, we further have

$$
x_{1}\left(t_{q}^{(n)}, \Phi_{n}\right) \geq x_{1}\left(s_{q}^{(n)}, \Phi_{n}\right) \exp \left[-\gamma_{0}\left(t_{q}^{(n)}-s_{q}^{(n)}\right)\right] .
$$

Consequently, by (3.12)

$$
\frac{\varepsilon}{n^{2}} \geq \frac{\varepsilon}{n} \exp \left[-\gamma_{0}\left(t_{q}^{(n)}-s_{q}^{(n)}\right)\right] \text {. }
$$

Hence,

$$
t_{q}^{(n)}-s_{q}^{(n)} \geq \frac{\ln n}{\gamma_{0}} \text { for all } q>K_{1}^{(n)} .
$$

By (3.9), there is constant $P>\lambda$ such that

$$
\int_{t}^{t+\beta}\left[r_{1}(s)-b_{1}(s) \varepsilon-\frac{a_{1}(s)}{k_{1}(s)}\left(x_{2}^{*}\left(s-\tau_{2}\right)+\varepsilon_{1}\right)\right] \mathrm{d} s>\delta
$$

for all $t \geq T_{2}$ and $\beta \geq P$.

Let $\bar{x}_{2 \varepsilon}(t)$ be the solution of system (3.10) with initial value $\bar{x}_{2 \varepsilon}\left(s_{q}^{(n)}\right)=x_{2}\left(s_{q}^{(n)}, \Phi_{n}\right)$. Since for any $n, q$ and $t \in\left[s_{q}^{(n)}, t_{q}^{(n)}\right]$ we have $x_{1}\left(t, X_{n}\right) \leq \varepsilon$ and

$$
\frac{\mathrm{d} x_{2}(t)}{\mathrm{d} t} \leq x_{2}(t)\left[r_{2}(t)-\frac{a_{2}(t) x_{2}(t)}{\varepsilon+k_{2}(t)} \exp \left(-r_{2}^{u} \tau_{4}\right)\right],
$$

by the comparison theorem, we have

$$
x_{2}\left(t, \Phi_{n}\right) \leq \bar{x}_{2 \varepsilon}(t) \text { for all } t \in\left[s_{q}^{(n)}, t_{q}^{(n)}\right] .
$$

Since $\lim _{n \rightarrow \infty} s_{q}^{(n)}=\infty$ and Theorem 3.2, there is constant $K_{2}^{(n)}>K_{1}^{(n)}$ for every $n$ such that $x_{2}\left(s_{q}^{(n)}+\tau, \Phi_{n}\right) \leq M$ for all $q \geq K_{2}^{(n)}$. By the comparison theorem and $x_{2 \varepsilon}(t)$ is the globally uniformly attractive solution of system (3.10), we obtain that there is a constant $T_{3} \geq T_{2}$ and such that

$$
\bar{x}_{2 \varepsilon}(t)<x_{2 \varepsilon}(t)+\frac{\varepsilon_{1}}{2} \text { for all } t>T_{3}+s_{q}^{(n)}+\tau .
$$

By (3.14), there is an integer $N_{0} \geq N_{1}$ such that

$$
t_{q}^{(n)}-s_{q}^{(n)}>T_{3}+\tau+P \text { for all } n>N_{0}, q>K_{2}^{(n)} .
$$

Further, by (3.11), (3.16) and (3.17) we have

$$
x_{2}\left(t, \Phi_{n}\right)<x_{20}^{*}(t)+\varepsilon_{1}
$$

for all $t \in\left[s_{q}^{(n)}+T_{3}+\tau, t_{q}^{(n)}\right]$ and $n \geq N_{0}$. Hence, when $n \geq N_{0}$ and $q \geq K^{(n)}$, integrating the first equation of system (1.2) from $s_{q}^{(n)}+T_{3}+\tau$ to $t_{q}^{(n)}$, by (3.12), (3.13), (3.15), and (3.18) we have

$$
\begin{aligned}
& x_{1}\left(t_{q}^{(n)}, \Phi_{n}\right)=x_{1}\left(s_{q}^{(n)}+T_{3}+\tau, \Phi_{n}\right) \\
& \times \exp \int_{s_{q}^{(n)} t_{+T_{3}+\tau}^{(n)}}^{t_{q}}\left[r_{1}(t)-b_{1}(t) x_{1}\left(t, \Phi_{n}\right)-\frac{a_{1}(t) x_{2}\left(t, \Phi_{n}\right)}{x_{1}\left(t, \Phi_{n}\right)+k_{1}(t)}\right] \mathrm{d} t \\
& \geq \frac{\varepsilon}{n^{2}} \exp \int_{s_{q}^{(n)}+T_{3}+\tau}^{t_{q}^{(n)}}\left[r_{1}(t)-b_{1}(t) \varepsilon-\frac{a_{1}(t)\left(x_{20}^{*}(t)+\varepsilon_{1}\right)}{k_{1}(t)}\right] \mathrm{d} t[8 p t] \\
& >\frac{\varepsilon}{n^{2}} .
\end{aligned}
$$


This leads to a contradiction with (3.12). Therefore, Claim 3.2 is true.

Finally, from Claims 3.1 and 3.2 we see that Theorem 3.3 is proved and this completes the proof of this theorem.

Remark 3.1 Nindjin and Aziz-AlaouiIn [11] discussed the following system

$$
\left\{\begin{array}{l}
\frac{\mathrm{d} x_{1}(t)}{\mathrm{d} t}=x_{1}(t)\left[r_{1}-b_{1} x_{1}(t)-\frac{a_{1} x_{2}(t)}{x_{1}(t)+k_{1}}\right] \\
\frac{\mathrm{d} x_{2}(t)}{\mathrm{d} t}=x_{2}(t)\left[r_{2}-\frac{a_{2} x_{2}(t-\tau)}{x_{1}(t-\tau)+k_{2}}\right]
\end{array} .\right.
$$

They stated that if

$$
r_{2} a_{1}\left(\frac{r_{1}}{b_{1}}+k_{2}\right) e^{r_{2} \tau}<r_{1} k_{1} a_{2},
$$

then system (3.19) is permanent. We note that, when system (1.2) degenerates into system (3.19), the condition (3.20) clearly implies the condition (3.8) in Theorem 3.3. So the theorem of A. F. Nindjin, M. Aziz-Alaouiln (Theorem 5 in [11]) is a special case of Theorem 3.3. So our results are fresh and more general.

A direct consequence of Theorem 3.3 is the following result on the permanence of system (3.3) and (3.4).

Corollary 3.2 Suppose that Assumptions $\left(\mathrm{H}_{1}\right)-\left(\mathrm{H}_{3}\right)$ hold and there is a constant $\lambda>0$ such that

$$
\liminf _{t \rightarrow \infty} \int_{t}^{t+\lambda}\left[r_{1}(s)-\frac{a_{1}(s)}{k_{1}(s)} x_{2}^{*}(s)\right], \mathrm{d} \tau>0
$$

where $x_{2}^{*}(t)$ is be any solution of the following system

$$
\frac{\mathrm{d} x_{2}(t)}{\mathrm{d} t}=x_{2}(t)\left[r_{2}(t)-\frac{a_{2}(t) x_{2}(t)}{k_{2}(t)}\right] \text {, }
$$

then system (3.3) is permanent.

Finally, we proceed to the discussion global stability of any positive solution of system (1.2). We first derive certain upperbound estimates for solution of system (1.2).

Theorem 3.4 Let $x(t)=\left(x_{1}(t), x_{2}(t)\right)$ denote any solutions of system (1.2) corresponding to initial conditions (1.3). Suppose that Assumptions $\left(\mathrm{H}_{1}\right),\left(\mathrm{H}_{3}\right)$ hold, and $b_{1}^{l}>0, a_{2}^{l}>0$, then there is a constant $T>0$ such that if $t \geq T$,

$$
0<x_{1}(t) \leq M_{1}, 0<x_{2}(t) \leq M_{2},
$$

where

$$
M_{1}=\frac{r_{1}^{u} e^{r_{1}^{u} \tau}}{b_{1}^{l}}, M_{2}=\frac{r_{2}^{u} e^{r_{2}^{u} \tau}\left(M_{1}+k_{2}^{u}\right)}{a_{2}^{l}} .
$$

The proof of Theorem 3.4 is similar to that of Theo- rem 2.1 in [16], we therefore omit it here.

We now formulate the global stability of any positive solutions of system (1.2).

Theorem 3.5 Let $x^{*}(t)=\left(x_{1}^{*}(t), x_{2}^{*}(t)\right)$ denote any positive solutions of system (1.2). Suppose that Assumptions $\left(\mathrm{H}_{1}\right),\left(\mathrm{H}_{3}\right)$ hold, and $b_{1}^{l}>0, a_{2}^{l}>0$, assume further that

$$
\left(\mathrm{H}_{4}\right) \liminf _{t \rightarrow \infty} B_{i}(t)>0
$$

where

$$
\begin{aligned}
B_{1}(t)= & b_{1}(t)-\frac{a_{1}(t) M_{2}}{k_{1}^{2}(t)} \\
- & {\left[r_{2}(t)+b_{1}(t) M_{1}+\frac{a_{2}(t) M_{2}}{k_{1}(t)}\right] \int_{t}^{t+\tau_{1}} b_{1}(s) \mathrm{d} s } \\
- & {\left[b_{1}\left(t+\tau_{1}\right) M_{1}+\frac{a_{1}\left(t+\tau_{1}\right)}{k_{1}^{2}\left(t+\tau_{1}\right)} M_{1} M_{2}\right] \int_{t+\tau_{1}}^{t+2 \tau_{1}} b_{1}(s) \mathrm{d} s } \\
- & \frac{a_{2}\left(t+\tau_{3}\right) M_{2}}{k_{2}^{2}\left(t+\tau_{3}\right)}-\frac{a_{2}\left(t+\tau_{3}\right) M_{2}^{2}}{k_{2}^{2}\left(t+\tau_{3}\right)} \int_{t+\tau_{3}}^{t+\tau_{4}} \frac{a_{2}(s)}{k_{2}(s)} \mathrm{d} s \\
B_{2}(t)= & \frac{a_{2}(t)}{M_{1}+k_{2}(t)}-\left[r_{2}(t)+\frac{a_{2}(t) M_{2}}{k_{2}(t)} \int_{t}^{t+\tau_{4}} \frac{a_{2}(s)}{k_{2}(s)} \mathrm{d} s\right. \\
& -\frac{a_{1}\left(t+\tau_{2}\right)}{k_{1}\left(t+\tau_{2}\right)}-\frac{a_{2}\left(t+\tau_{4}\right) M_{2}}{k_{2}\left(t+\tau_{4}\right)} \int_{t+\tau_{4}}^{t+\tau_{4}} \frac{a_{2}(s)}{k_{2}(s)} \mathrm{d} s \\
& -\frac{a_{1}\left(t+\tau_{2}\right) M_{1}}{k_{1}\left(t+\tau_{2}\right)} \int_{t+\tau_{2}}^{t+\tau_{2}} b_{1}(s) \mathrm{d} s
\end{aligned}
$$

Then the solution $x^{*}(t)=\left(x_{1}^{*}(t), x_{2}^{*}(t)\right)$ is globally asymptotically stable.

Proof: Let $\left(x_{1}(t), x_{2}(t)\right)$ be any solution of system (1.2) and (1.3). It follows from Theorem 3.4 that there exist positive constants $T$ and $M_{i}$ (defined by (3.21), such that for all $t \geq T$,

$$
0<x_{i}(t) \leq M_{i}, 0<x_{i}^{*}(t) \leq M_{i}, i=1,2 .
$$

We define

$$
V_{11}(t)=\left|\ln x_{1}^{*}(t)-\ln x_{1}(t)\right| .
$$

Calculating the upper right derivative of $V_{11}(t)$ along solutions of system (1.2), it follows that

$$
\begin{aligned}
D^{+} V_{11}(t)= & \operatorname{sgn}\left(x_{1}^{*}(t)-x_{1}(t)\right)\left[\frac{\dot{x}_{1}^{*}(t)}{x_{1}^{*}(t)}-\frac{\dot{x}_{1}(t)}{x_{1}(t)}\right] \\
= & \operatorname{sgn}\left(x_{1}^{*}(t)-x_{1}(t)\right) \\
& \times\left\{-\left[\frac{a_{1}(t) x_{2}^{*}\left(t-\tau_{2}\right)}{x_{1}^{*}\left(t-\tau_{1}\right)+k_{1}(t)}-\frac{a_{1}(t) x_{2}\left(t-\tau_{2}\right)}{x_{1}\left(t-\tau_{1}\right)+k_{1}(t)}\right]\right. \\
& \left.-b_{1}(t)\left[x_{1}^{*}\left(t-\tau_{1}\right)-x_{1}\left(t-\tau_{1}\right)\right]\right\}
\end{aligned}
$$




$$
\begin{aligned}
= & \operatorname{sgn}\left(x_{1}^{*}(t)-x_{1}(t)\right)\left\{-b_{1}(t)\left[x_{1}^{*}\left(t-\tau_{1}\right)-x_{1}\left(t-\tau_{1}\right)\right]\right. \\
& -\frac{a_{1}(t)}{\Delta_{1}(t)}\left[k_{1}(t)\left[x_{2}^{*}\left(t-\tau_{2}\right)-x_{2}\left(t-\tau_{2}\right)\right]\right. \\
& +x_{1}\left(t-\tau_{1}\right)\left[x_{2}^{*}\left(t-\tau_{2}\right)-x_{2}\left(t-\tau_{2}\right)\right] \\
& \left.\left.-x_{2}\left(t-\tau_{2}\right)\left[x_{1}^{*}\left(t-\tau_{1}\right)-x_{1}\left(t-\tau_{1}\right)\right]\right]\right\} \\
= & \operatorname{sgn}\left(x_{1}^{*}(t)-x_{1}(t)\right)\left\{\left[-b_{1}(t)+\frac{a_{1}(t) x_{2}\left(t-\tau_{2}\right)}{\Delta_{1}(t)}\right]\right. \\
& \times\left[x_{1}^{*}\left(t-\tau_{1}\right)-x_{1}\left(t-\tau_{1}\right)\right]-\frac{a_{1}(t)}{x_{1}^{*}\left(t-\tau_{1}\right)+k_{1}(t)} \\
& \left.\times\left[x_{2}^{*}\left(t-\tau_{2}\right)-x_{2}\left(t-\tau_{2}\right)\right]\right\} \\
\leq & -\left[b_{1}(t)-\frac{a_{1}(t) x_{2}\left(t-\tau_{2}\right)}{\Delta_{1}(t)}|| x_{1}^{*}(t)-x_{1}(t) \mid\right. \\
& +b_{1}(t)\left|\int_{t-\tau_{1}}^{t}\left[\dot{x}_{1}^{*}(u)-\dot{x}_{1}(u)\right] \mathrm{d} u\right| \\
& +\frac{a_{1}(t)}{k_{1}(t)}\left|x_{2}^{*}\left(t-\tau_{2}\right)-x_{2}\left(t-\tau_{2}\right)\right|
\end{aligned}
$$

where

$$
\begin{gathered}
\Delta_{1}(t)=\left[x_{1}^{*}\left(t-\tau_{1}\right)+k_{1}(t)\right]\left[x_{1}\left(t-\tau_{1}\right)+k_{1}(t)\right] \\
\Delta_{2}(t)=\left[x_{1}^{*}\left(t-\tau_{3}\right)+k_{2}(t)\right]\left[x_{1}\left(t-\tau_{3}\right)+k_{2}(t)\right] .
\end{gathered}
$$

On substituting (1.2) into (3.24), we derive that

$$
\begin{aligned}
& D^{+} V_{11}(t) \\
\leq & -\left[b_{1}(t)-\frac{a_{1}(t) x_{2}\left(t-\tau_{2}\right)}{\Delta_{1}(t)}\right]\left|x_{1}^{*}(t)-x_{1}(t)\right| \\
& +\frac{a_{1}(t)}{k_{1}(t)}\left|x_{2}^{*}\left(t-\tau_{2}\right)-x_{2}\left(t-\tau_{2}\right)\right| \\
& +b_{1}(t) \mid \int_{t-\tau_{1}}^{t}\left\{\left[r_{1}(u) x_{1}^{*}(u)-b_{1}(u) x_{1}^{*}(u) x_{1}^{*}\left(u-\tau_{1}\right)\right.\right. \\
& \left.-\frac{a_{1}(u) x_{1}^{*}(u) x_{2}^{*}\left(u-\tau_{2}\right)}{x_{1}^{*}\left(u-\tau_{1}\right)+k_{1}(u)}\right] \\
& -\left[r_{1}(u) x_{1}(u)-b_{1}(u) x_{1}(u) x_{1}\left(u-\tau_{1}\right)\right. \\
& \left.\left.-\frac{a_{1}(u) x_{1}(u) x_{2}\left(u-\tau_{2}\right)}{x_{1}\left(u-\tau_{1}\right)+k_{1}(u)}\right]\right\} \mathrm{d} u \\
\leq & -\left[b_{1}(t)-\frac{a_{1}(t) x_{2}\left(t-\tau_{2}\right)}{\Delta_{1}(t)}|| x_{1}^{*}(t)-x_{1}(t) \mid\right. \\
& +\frac{a_{1}(t)}{k_{1}(t)}\left|x_{2}^{*}\left(t-\tau_{2}\right)-x_{2}\left(t-\tau_{2}\right)\right|
\end{aligned}
$$

$$
\begin{aligned}
& +b_{1}(t) \mid \int_{t-\tau_{1}}^{t}\left\{\left[r_{1}(u)+b_{1}(u) x_{1}\left(u-\tau_{1}\right)\right.\right. \\
& +\frac{a_{1}(u)}{\Delta_{1}(u)}\left[k_{1}(u) x_{2}\left(u-\tau_{2}\right)\right. \\
& \left.\left.+x_{1}\left(u-\tau_{1}\right) x_{2}^{*}\left(u-\tau_{2}\right)\right]\right]\left|x_{1}^{*}(u)-x_{1}(u)\right| \\
& +\left[b_{1}(u) x_{1}^{*}(u)+\frac{a_{1}(u)}{\Delta_{1}(u)} x_{1}(u) x_{2}\left(u-\tau_{2}\right)\right] \\
& \times\left|x_{1}^{*}\left(u-\tau_{1}\right)-x_{1}\left(u-\tau_{1}\right)\right| \\
& +\frac{a_{1}(u)}{\Delta_{1}(u)}\left[k_{1}(u) x_{1}^{*}(u)+x_{1}(u) x_{1}\left(u-\tau_{1}\right)\right] \\
& \left.\times\left|x_{2}^{*}\left(u-\tau_{2}\right)-x_{2}\left(u-\tau_{2}\right)\right|\right\} \mathrm{d} u .
\end{aligned}
$$

\section{Define}

$$
\begin{aligned}
V_{12}(t)= & \int_{t}^{t+\tau_{1}} \int_{s-\tau_{1}}^{t} b_{1}(s)\left\{\left[r_{1}(u)+b_{1}(u) x_{1}\left(u-\tau_{1}\right)\right.\right. \\
& +\frac{a_{1}(u)}{\Delta_{1}(u)}\left[k_{1}(u) x_{2}\left(u-\tau_{2}\right)\right. \\
& \left.\left.+x_{1}\left(u-\tau_{1}\right) x_{2}^{*}\left(u-\tau_{2}\right)\right]\right]\left|x_{1}^{*}(u)-x_{1}(u)\right| \\
& +\left[b_{1}(u) x_{1}^{*}(u)+\frac{a_{1}(u)}{\Delta_{1}(u)} x_{1}(u) x_{2}\left(u-\tau_{2}\right)\right] \\
& \times\left|x_{1}^{*}\left(u-\tau_{1}\right)-x_{1}\left(u-\tau_{1}\right)\right|+\frac{a_{1}(u)}{\Delta_{1}(u)}\left[k_{1}(u) x_{1}^{*}(u)\right. \\
& \left.\left.+x_{1}(u) x_{1}\left(u-\tau_{1}\right)\right]\left|x_{2}^{*}\left(u-\tau_{2}\right)-x_{2}\left(u-\tau_{2}\right)\right|\right\} \mathrm{d} u \mathrm{~d} s .
\end{aligned}
$$

We obtain from (3.25) and (3.26) that

$$
\begin{aligned}
& D^{+} V_{11}(t)+\dot{V}_{12}(t) \\
& \leq-\left[b_{1}(t)-\frac{a_{1}(t) x_{2}\left(t-\tau_{2}\right)}{\Delta_{1}(t)}\right]\left|x_{1}^{*}(t)-x_{1}(t)\right| \\
&+\frac{a_{1}(t)}{k_{1}(t)}\left|x_{2}^{*}\left(t-\tau_{2}\right)-x_{2}\left(t-\tau_{2}\right)\right| \\
&+\int_{t}^{t+\tau_{1}} b_{1}(s) \mathrm{ds}\left\{\left[r_{1}(t)+b_{1}(t) x_{1}\left(t-\tau_{1}\right)\right.\right. \\
&\left.+\frac{a_{1}(t)}{\Delta_{1}(t)}\left[k_{1}(t) x_{2}\left(t-\tau_{2}\right)+x_{1}\left(t-\tau_{1}\right) x_{2}^{*}\left(t-\tau_{2}\right)\right]\right] \\
& \times\left|x_{1}^{*}(t)-x_{1}(t)\right|+\left[b_{1}(t) x_{1}^{*}(t)+\frac{a_{1}(t)}{\Delta_{1}(t)} x_{1}(t) x_{2}\left(t-\tau_{2}\right)\right] \\
& \times\left|x_{1}^{*}\left(t-\tau_{1}\right)-x_{1}\left(t-\tau_{1}\right)\right|+\frac{a_{1}(t)}{\Delta_{1}(t)}\left[k_{1}(t) x_{1}^{*}(t)\right. \\
&\left.\left.+x_{1}(t) x_{1}\left(t-\tau_{1}\right)\right]\left|x_{2}^{*}\left(t-\tau_{2}\right)-x_{2}\left(t-\tau_{2}\right)\right|\right\} .
\end{aligned}
$$


We now define

$$
V_{1}(t)=V_{11}(t)+V_{12}(t)+V_{13}(t)
$$

where

$$
\begin{aligned}
V_{13}(t)= & \int_{t-\tau_{1}}^{t} \int_{l+\tau_{1}}^{l+2 \tau_{1}} b_{1}(s)\left[b_{1}\left(l+\tau_{1}\right) M_{1}+\frac{a_{1}\left(l+\tau_{1}\right)}{k_{1}^{2}\left(l+\tau_{1}\right)} M_{1} M_{2}\right] \\
& \times\left|x_{1}^{*}(l)-x_{1}(l)\right| \mathrm{d} s \mathrm{~d} l
\end{aligned}
$$

and

$$
\begin{aligned}
V_{14}(t) & =\int_{t-\tau_{2}}^{t} \frac{a_{1}\left(s+\tau_{2}\right)}{k_{1}\left(s+\tau_{2}\right)}\left|x_{2}^{*}(s)-x_{2}(s)\right| \mathrm{d} s \\
& +\int_{t-\tau_{2}}^{t} \int_{l+\tau_{2}}^{l+\tau_{1}+\tau_{2}} b_{1}(s) \frac{a_{1}\left(l+\tau_{2}\right) M_{1}}{k_{1}\left(l+\tau_{2}\right)}\left|x_{2}^{*}(l)-x_{2}(l)\right| \mathrm{d} s \mathrm{~d} l .
\end{aligned}
$$

It then follows from (3.23) and (3.27)-(3.30) that for $t \geq T+\tau$,

$$
\begin{aligned}
& D^{+} V_{1}(t) \\
& \leq-\left\{b_{1}(t)-\frac{a_{1}(t) M_{2}}{k_{1}^{2}(t)}-\left[r_{1}(t)+b_{1}(t) M_{1}+\frac{a_{1}(t) M_{2}}{k_{1}(t)}\right]\right. \\
& \quad \times \int_{t}^{t+\tau_{1}} b_{1}(s) \mathrm{d} s-\left[b_{1}\left(t+\tau_{1}\right) M_{1}+\frac{a_{1}\left(t+\tau_{1}\right)}{k_{1}^{2}\left(t+\tau_{1}\right)} M_{1} M_{2}\right] \\
& \left.\quad \times \int_{t+\tau_{1}}^{t+2 \tau_{1}} b_{1}(s) \mathrm{d} s\right\}\left|x_{1}^{*}(t)-x_{1}(t)\right|+\left[\frac{a_{1}\left(t+\tau_{2}\right) M_{1}}{k_{1}\left(t+\tau_{2}\right)}\right. \\
& \left.\quad \times \int_{t+\tau_{2}}^{t+\tau_{1}+\tau_{2}} b_{1}(s) \mathrm{d} s+\frac{a_{1}\left(t+\tau_{2}\right)}{k_{1}\left(t+\tau_{2}\right)}\right]\left|x_{2}^{*}(t)-x_{2}(t)\right| .
\end{aligned}
$$

Similarly, we define

$$
V_{2}(t)=V_{21}(t)+V_{22}(t)
$$

where

$$
V_{21}(t)=\left|\ln x_{2}^{*}(t)-\ln x_{2}(t)\right|
$$

and

$$
\begin{aligned}
V_{22}(t)= & \int_{t}^{t+\tau_{4}} \int_{s-\tau_{4}}^{t} \frac{a_{2}(s)}{k_{2}(s)}\left\{\left[r_{2}(u)+\frac{a_{2}(u) M_{2}}{x_{1}^{*}\left(u-\tau_{3}\right)+k_{2}(u)}\right]\right. \\
& \times\left|x_{2}^{*}(u)-x_{2}(u)\right|+\frac{a_{2}(u) M_{2}}{x_{1}^{*}\left(u-\tau_{3}\right)+k_{2}(u)} \\
& \times\left|x_{2}^{*}\left(u-\tau_{4}\right)-x_{2}\left(u-\tau_{4}\right)\right| \\
& \left.+\frac{a_{2}(u) M_{2}^{2}}{\Delta_{2}(u)}\left|x_{1}^{*}\left(u-\tau_{3}\right)-x_{1}\left(u-\tau_{3}\right)\right|\right\} \mathrm{d} u \mathrm{~d} s .
\end{aligned}
$$

Calculating the upper right derivative of $V_{2}(t)$ along solutions of system (1.2), we derive for $t \geq T+\tau$ that

$$
\begin{aligned}
& D^{+} V_{2}(t) \\
& \leq-\left\{\frac{a_{2}(t)}{M_{1}+k_{2}(t)}-\left[r_{2}(t)+\frac{a_{2}(t) M_{2}}{k_{2}(t)}\right]\right. \\
& \left.\quad \times \int_{t}^{t+\tau_{4}} \frac{a_{2}(s)}{k_{2}(s)} \mathrm{d} s\right\}\left|x_{2}^{*}(t)-x_{2}(t)\right| \\
& +\left[\frac{a_{2}(t) M_{2}}{k_{2}^{2}(t)}+\frac{a_{2}(t) M_{2}^{2}}{k_{2}^{2}(t)} \int_{t}^{t+\tau_{4}} \frac{a_{2}(s)}{k_{2}(s)} \mathrm{d} s\right] \\
& \times\left|x_{1}^{*}\left(t-\tau_{3}\right)-x_{1}\left(t-\tau_{3}\right)\right|+\frac{a_{2}(t) M_{2}}{k_{2}(t)} \\
& \quad \times \int_{t}^{t+\tau_{4}} \frac{a_{2}(s)}{k_{2}(s)} \mathrm{d} s\left|x_{2}^{*}\left(t-\tau_{4}\right)-x_{2}\left(t-\tau_{4}\right)\right| .
\end{aligned}
$$

We define a Lyapunov functional $V(t)$ as

$$
V(t)=V_{1}(t)+V_{2}(t)+V_{3}(t)+V_{4}(t),
$$

where

$$
\begin{aligned}
V_{3}(t)= & \int_{t-\tau_{3}}^{t} \frac{a_{2}\left(s+\tau_{3}\right) M_{2}}{k_{2}^{2}\left(s+\tau_{3}\right)}\left|x_{1}^{*}(s)-x_{1}(s)\right| \mathrm{d} s \\
& +\int_{t-\tau_{3}}^{t} \int_{l+\tau_{3}}^{l+\tau_{3}+\tau_{4}} \frac{a_{2}(s)}{k_{2}(s)} \frac{a_{2}\left(l+\tau_{3}\right) M_{2}^{2}}{k_{2}^{2}\left(l+\tau_{3}\right)}\left|x_{1}^{*}(l)-x_{1}(l)\right| \mathrm{d} s \mathrm{~d} l
\end{aligned}
$$

and

$$
V_{4}(t)=\int_{t-\tau_{4}}^{t} \int_{l+\tau_{4}}^{l+2 \tau_{4}} \frac{a_{2}(s)}{k_{2}(s)} \frac{a_{2}\left(l+\tau_{4}\right) M_{2}}{k_{2}\left(l+\tau_{4}\right)}\left|x_{2}^{*}(l)-x_{2}(l)\right| \mathrm{d} s \mathrm{~d} l .
$$

It then follows from (3.22), (3.31), and (3.35)-(3.38) that for $t \geq T+\tau$

$$
D^{+} V(t) \leq-B_{1}(t)\left|x_{1}^{*}(t)-x_{1}(t)\right|-B_{2}(t)\left|x_{2}^{*}(t)-x_{2}(t)\right| \text {. }
$$

where $B_{1}(t)$ and $B_{2}(t)$ are defined in (3.22).

By Assumption $\left(\mathrm{H}_{4}\right)$, there exist positive constants $\alpha_{1}, \quad \alpha_{2}$ and $T^{*} \geq T+\tau$ such that if $t \geq T^{*}$

$$
B_{i}(t) \geq \alpha_{i}>0, \quad i=1,2 .
$$

Integrating both sides of (3.39) on interval $\left[T^{*}, t\right]$,

$$
V(t)+\sum_{i=1}^{2} \int_{T^{*}}^{t} B_{i}(s)\left|x_{i}^{*}(s)-x_{i}(s)\right| \mathrm{d} s \leq V\left(T^{*}\right)
$$

It follows from (3.40) and (3.41) that 
$V(t)+\sum_{i=1}^{2} \alpha_{i} \int_{T^{*}}^{t}\left|x_{i}^{*}(s)-x_{i}(s)\right| \mathrm{d} s \leq V\left(T^{*}\right)$ for all $t \geq T^{*}$. Therefore, $V(t)$ is bounded on $\left[T^{*}, \infty\right]$ and also

$$
\int_{T^{*}}^{\infty}\left|x_{i}^{*}(s)-x_{i}(s)\right| \mathrm{d} s<\infty, \quad i=1,2 .
$$

By Theorem 3.4, $\left|x_{i}^{*}(t)-x_{i}(t)\right|(i=1,2)$ are bounded on $\left[T^{*}, \infty\right]$.

On the other hand, it is easy to see that $\dot{x}_{i}^{*}(t)$ and $\dot{x}_{i}(t)(i=1,2)$ are bounded for $t \geq T^{*}$. Therefore, $\left|x_{i}^{*}(t)-x_{i}(t)\right|(i=1,2)$ are uniformly continuous on $\left[T^{*}, \infty\right]$. By Barbalat's Lemma ([17], Lemmas 1.2.2 and 1.2.3), we conclude that

$$
\lim _{t \rightarrow \infty}\left|x_{i}^{*}(t)-x_{i}(t)\right|=0, \quad i=1,2 .
$$

This completes the proof of this theorem.

Remark 3.2 If time delays $\tau_{1}, \tau_{2}, \tau_{3}, \tau_{3}$ and $\tau_{4}$ are naturally subject to fluctuation in time in system (1.2). Similar Theorem 3.1-3.5, we can obtain the sufficient conditions on the permanence and globally asymptotically stable of any positive solutions for system (1.2).

Finally, we give some examples to illustrate the feasibility of our main results on the permanence of system (1.2).

Example 3.1 In system (1.2), let $r_{1}=0.2 e^{0.2}+0.05$ $+0.1 \sin t, \quad b_{1}=0.9 \cos t, \quad r_{2}=0.4, \quad a_{1}=a_{2}=1, k_{1}=2$, $\tau_{1}=0.1, \tau_{2}=0.5, k_{2}=1$. It is easy to verify that coefficients of system (1.2) satisfy (3.8). By Theorem 3.2 and 3.3, system (1.2) is permanent.

Example 3.2 In system (1.2), let $r_{1}=0.2 e^{0.2}$, $b_{1}=0.1, \quad a_{1}=a_{2}=1, \quad k_{1}=2, r_{2}=0.4, \tau_{1}=0.1$, $\tau_{2}=0.5, k_{2}=1$. By Theorem 3.2 we see that the component $x_{2}$ of system (1.2) is permanent. However, it is easy verify that

$$
r_{1}-a_{1} r_{2} k_{2} e^{r_{2} \tau_{4}} / k_{1} a_{2}=0.2 e^{0.2}-0.4 e^{0.2} / 2 \equiv 0
$$

thus (3.8) does not hold for system (1.2) and we cannot get any information by Theorem 3.3. In this case, we note that $r_{1}-a_{1} r_{2} k_{2} / k_{1} a_{2}=0.2 e^{0.2}-0.4 / 2>0$ and numerical simulation suggests that system (1.2) with a sequence initial condition $\left(\phi_{1}, \phi_{2}\right)$ is permanent.

In the example 3.2, from numerical simulation, we note that the time delays are harmless for the permanence. Therefore, as an improvement of Theorems 3.2 and 3.3, we give the following interesting conjecture.

Conjecture: Suppose the assumptions of Corollary 3.2 hold, then system (1.2) is permanent.

\section{Acknowledgements}

This work was supported by the National Natural Science Foundation of P.R. China (11001235, 10961022) and the Natural Science Foundation of Xinjiang University (BS100104, BS080105).

\section{References}

[1] M. Fan, Q. Wang and X. F. Zou, "Dynamics of Nonautonomous Ratio-Dependent Predator-Prey System," Proceedings of the Royal Society of Edinburgh: Section A, Vol. 133, No. 1, 2003, pp. 97-118. doi:10.1017/S0308 210500002304

[2] Y. Kuang, "Delay Differential Equations, with Applications in Population Dynamics," Academic Press, New York, 1993.

[3] Z. Teng, "Persistence and Stability in General Non-autonomous Single-Species Kolmogorov Systems with Delays,” Nonlinear Analysis: Real World Applications, Vol. 8, No. 1, 2007, pp. 230-248. doi:10.1016/j.nonrwa. 2005.08.003

[4] Z. Teng and M. Rehim, "Persistence in Nonautonomous Predator-Prey Systems with Infinite Delays,” Journal of Computational and Applied Mathematics, Vol. 197, No. 2, 2006, pp. 302-321. doi:10.1016/j.cam.2005.11.006

[5] R. K. Upadhyay and S. R. K. Iyengar, "Effect of Seasonality on the Dynamics of 2 and 3 Species Prey-Predator System," Nonlinear Analysis: Real World Applications, Vol. 6, No. 3, 2005, pp. 509-530. doi:10.1016/j.nonrwa. 2004.11.001

[6] R. Xu, M. A. J. Chaplain and F. A. Davidson, "Periodic Solutions for a Predator-Prey Model with Holling-Type Functional Response and Time Delays,” Applied Mathematics and Computation, Vol. 161, No. 2, 2005, pp. $637-$ 654. doi:10.1016/j.amc.2003.12.054

[7] M. A. Aziz-Alaoui, "Study of a Leslie-Gower-Type Tritrophic Population,” Chaos, Solitons \& Fractals, Vol. 14, No. 8, 2002, pp. 1275-1293. doi:10.1016/S0960-0779(02) 00079-6

[8] E. Beretta and Y. Kuang, "Global Analyses in Some Delayed Ratio-Depended Predator-Prey Systems," Nonlinear Analysis: Theory, Methods \& Applications, Vol. 32, No. 3, 1998, pp. 381-408.

[9] C. Letellier and M. A. Aziz-Alaoui, "Analysis of the Dynamics of a Realistic Ecological Model,” Chaos, Solitons \& Fractals, Vol. 13, No. 1, 2002, pp. 95-107. doi:10.1016/S0960-0779(00)00239-3

[10] M. A. Aziz-Alaoui and M. D. Okiye, "Boundedness and Global Stability for a Predator-Prey Model with Modified Leslie-Gower and Holling-Type II Schemes," Applied Mathematics Letters, Vol. 16, No. 7, 2003, pp. 1069-1075. doi:10.1016/S0893-9659(03)90096-6

[11] A. F. Nindjin and M. A. Aziz-Alaoui, "Analysis of a Predator-Prey Model with Modified Leslie-Gower and Holling-Type II Schemes with Time Delay,” Nonlinear Analysis: Real World Applications, Vol. 7, No. 5, 2006, pp. 1104-1118. doi:10.1016/j.nonrwa.2005.10.003

[12] R. K. Upadhyay and V. Rai, "Crisis-Limited Chaotic Dynamics in Ecological Systems,” Chaos, Solitons \& Fractals, Vol. 12, No. 2, 2001, pp. 205-218. doi:10.1016/ 
S0960-0779(00)00141-7

[13] R. R. Vance and E. A. Coddington, "A Nonautonomous Model of Population Growth,” Journal of Mathematical Biology, Vol. 27, No. 5, 1989, pp. 491-506. doi:10.1007/ BF00288430

[14] Z. Teng and Z. Li, "Permanence Criteria in Non-autonomous Predator-Prey Kolmogorov Systems and Its Applications,” Dynamical Systems, Vol. 19, No. 2, 2004, pp. 171-194. doi:10.1080/14689360410001698851

[15] Z. Teng and L. Chen, "Uniform Persistence and Existence of Strictly Positive Solutions in Nonautonomous
Lotka-Volterra Competitive Systems with Delays,” Computers \& Mathematics with Applications, Vol. 37, No. 7, 1999, pp. 61-71.

[16] W. Wang and Z. Ma. "Harmless Delays for Uniform Persistence," Journal of Mathematical Analysis and Applications, Vol. 158, No. 1, 1991, pp. 256-268. doi:10. 1016/0022-247X(91)90281-4

[17] K. Gopalsamy, "Stability and Oscillations in Delay Different Equations of Population Dynamics,” Kluwer Academic, Norwell, 1992. 